\title{
Evangelizar la cibercultura: los retos de la ciberteología
}

\author{
CARLOS ARBOLEDA MORA* \\ Universidad Pontificia Bolivariana (Colombia) \\ carlos.arboleda@upb.edu.co
}

\begin{abstract}
Resumen
Las nuevas tecnologías de la información van creando una nueva cultura que se denomina como cibercultura o ambiente digital, que incluye las tecnologías, las formas sociales de la producción tecnológica y las nuevas simbolizaciones y metáforas de la existencia de los hombres. Se presentan así algunos interrogantes fundamentales de inicio: ¿Cómo debe la iglesia responder a la nueva cultura virtual o cibercultura?, ¿Cómo se reflexiona teológicamente sobre la cibercultura?, ¿Cómo puede la iglesia usar el ciberespacio para hacerlo?, ¿Qué se está haciendo en este campo de la ciberteología? Se analizan las posibilidades y límites de una reflexión llamada ciberteología, con base epistémica y comunidad académica todavía incipientes, pero que con una teología colaborativa puede aportar a la reflexión teológica del siglo XXI donde hay problemas graves para resolver desde la fe, como son la transmisión de la experiencia, la corporalidad y la presencia, el desafío a la autoridad y la solidaridad.
\end{abstract}

Palabras clave: ciberteología, cibercultura, ciberevangelización, ambiente digital, ciberreligión.

\section{Evangelize cyberculture: the challenges of cybertheology}

\begin{abstract}
The new technologies of the information are creating a new culture that is known as cyberculture or digital environment, which includes the technologies, the social forms of the technological production and the new symbolizations and metaphors of the existence of the bumankind. There are some fundamental questions in beginning: How should the church respond to the new virtual culture or cyber-culture? How we reflect theologically on cyber-culture?, how can the church use cyberspace to do evangelization?, What it is doing in this field of the cyber-theology?. There are analyzed the possibilities and limits of a reflection called cyber-theology with epistemic bases and still emerging academic community, but that with a collaborative theology can reach to one theological reflection for the 21 st century where there are serious problems to solve in the believing community, such as the transmission of the experience, the body and the flesh, the challenge to the authority, the physicality and the presence, and solidarity.
\end{abstract}

Key words: cyber-theology, cyber-culture, cyber-evangelization, digital environment, cyber-religion

* Doctor en filosofía, Magister en Historia, Magister en Ciencias sociales. Sus últimas publicaciones son: Experiencia y testimonio (2014), Belleza y mistica (2015), Profundidady cultura (2008). Profesor de la UPB.

El artículo es el resultado del proyecto de investigación Biblia e interculturalidad (Cidi 2017) del G.I. Teología, Religión y Cultura de la UPB- Medellín-Colombia.

Recibido: 21/Octubre/2016 - Aceptado: 3/Marzo/2017 


\section{INTRODUCCIÓN}

Un gran reto, aceptado ya por la Iglesia católica, es la evangelización en y por el mundo virtual. Reto que significa un esfuerzo continuado por entender lo que es la virtualidad y cómo se transmite el mensaje del evangelio a través de él. El acelerado desarrollo de las tecnologías de la información y de la comunicación al servicio de tantas actividades humanas, aprovechable también en las tareas de la Iglesia, se constituye en una oportunidad para proponer la Evangelización en ambientes virtuales buscando potenciar la formación de los agentes de pastoral en competencias propias de este ambiente para su desempeño como evangelizadores.

Estamos ante una verdadera revolución cultural. La invención de la máquina de vapor creó una revolución industrial, luego la electricidad y la automatización aceleraron los cambios en las mentalidades: forma de hacer, forma de creer y forma de relacionarse. Se introdujo un cambio enorme con el paulatino abandono de la época agraria para pasar a la sociedad urbana. Los procesos de automatización, aumento de la velocidad en ellos y nuevos estilos de vida dieron origen a lo que se llamó la sociedad del bienestar y de la calidad de vida. Ahora, las potencialidades de la tecnología se van desarrollando y dan origen a una cultura cibernética, cibercultura o cultura digital, que no sólo consiste en desarrollos tecnológicos, sino en una nueva manera de vivir, sentir, experimentar, comunicarse, con grandes problemas (pobreza, exclusión, violación de derechos, márgenes de rechazado) pero que se presenta como el ambiente existencial para los humanos actuales y del próximo futuro.

Habrá muchas cosas buenas y muchas cosas sombrías. Paul Mason (2016), autor del libro Poscapitalismo: Hacia un nuevo futuro trae la hipótesis de que el capitalismo como modelo económico ha llegado a su fin y debemos prepararnos para una transición a un nuevo sistema basado en las nuevas tecnologías y la reestructuración del trabajo. Dice que entre los factores que han impulsado el neoliberalismo económico y social dinero fiduciario, financiarización, desequilibrios globales y tecnología de la información, es esta última la que marca el desarrollo hacia el futuro.

El único factor positivo que contrasta con todos los negativos señalados hasta el momento es la revolución tecnológica, que fue un producto del neoliberalismo y ha continuado avanzando con fuerza incluso desafiando a la crisis económica. "La sociedad de la información - ha escrito el filósofo Luciano Floridi- ha sido creada por la tecnología de más rápido crecimiento de toda la historia. Ninguna generación anterior había estado expuesta a tan extraordinaria aceleración del poder de la técnica sobre la realidad, con los correspondientes cambios sociales y responsabilidades éticas que ese poder comporta" (Mason, 2016: 54). 
Entre esas responsabilidades éticas está la calidad de vida digna de grandes sectores sociales entendiendo esa calidad no sólo como bienestar económico sino también como bienestar social, democracia, participación, inclusión y respeto. Hay que pensar, sin embargo, que las luces de colores de un comunitarismo futuro y de una preocupación por el bienestar social provocados por la revolución tecnológica, no pueden ocultar esos problemas éticos, sumados al analfabetismo digital, al digital divide ${ }^{1}$, al hambre material, a la pobreza y al vacío espiritual. Como ejemplo se puede citar el influjo de las redes sociales en el reclutamiento de jóvenes europeos para las filas del Estado Islámico. Su conversión no se hace en lugares físicos como mezquitas o colegios, sino en la red de Internet, a través de las redes sociales (Facebook, Youtube, Twitter, Instagram, What's Up). Señala Massimo Leone (2014), que la lucha jihadista usa las nuevas tecnologías y las adapta a sus fines de extender el pensamiento fundamentalista con su persuasión religiosa y su incitación al combate. Concluye diciendo que, a través de la red, la retórica jihadista les ofrece un modo de vida con sentido que cambia las perspectivas existenciales de los jóvenes y que las sociedades occidentales ya no son capaces de ofrecer.

Por eso, hay varias preguntas que se deben pensar: ¿Cómo debe la Iglesia o las Iglesias responder a la nueva cultura virtual o cibercultura? ¿Cómo se reflexiona teológicamente sobre la cibercultura? ¿Cómo puede la iglesia usar el ciberespacio para hacer teología y evangelización? ¿Qué se está haciendo en este campo de la ciberteología?

La hipótesis que guía este trabajo es que si las iglesias (todas las iglesias cristianas) con sus laicos e instituciones responden a las oportunidades y desafíos que el ambiente virtual presenta, teniendo en cuenta sus aspectos críticos y problemáticos, se lograría una reflexión teológica renovada, se descubrirían nuevas metodologías pastorales y se lograría obtener un campo de trabajo fecundo para el siglo XXI.

\footnotetext{
1 La expresión brecha digital (digital divide) destaca el hecho de que tanto las personas como los grupos y las naciones deben tener acceso a las nuevas tecnologías para participar en los beneficios prometidos por la globalización y el desarrollo y no quedarse rezagados posteriormente. Juan Pablo II, manifestaba "que la brecha entre los beneficiarios de los nuevos medios de información y expresión, y los que hasta ahora no han tenido acceso a ellos, no se convierta en otra persistente fuente de desigualdad y discriminación" (Juan Pablo II, 1997: s/p). La Red Informática de la Iglesia en América Latina (RIIAL) dentro de sus funciones está la de impulsar las actividades y proyectos que favorezcan la disminución de la brecha digital facilitando el acceso a los servicios tecnológicos de formación de los sectores más pobres y desfavorecidos, además animar y apoyar y dinamizar la pastoral de la Comunicación eclesial en lo referente al mundo del ciberespacio y las TIC. (Bello, 2014).
} 


\section{LA ORIENTACIÓN DEL MAGISTERIO DE LA IGLESIA}

Ya desde la década de los 60 del pasado siglo, a partir del ambiente de renovación que suscitó en el mundo cristiano el Concilio Vaticano II (1962-1965), la Iglesia católica se hizo consciente de que era necesario "utilizar todos aquellos maravillosos inventos de la técnica que el ingenio humano, con la ayuda de Dios, ha extraído de las cosas creadas" (Inter Mirifica, 2000: 1), y en ese sentido declaró que:

[...] la Iglesia católica, para llevar la salvación a todos los hombres y, en consecuencia, urgida por la necesidad de evangelizar, considera que forma parte de su misión predicar el mensaje de salvación, con la ayuda, también, de los medios de comunicación social, y enseñar a los hombres su recto uso. A la Iglesia, pues, le corresponde el derecho originario de utilizar y poseer toda clase de medios de este género, en cuanto que sean necesarios o útiles para la educación cristiana y para toda su labor de salvación de las almas; a los sagrados Pastores les compete la tarea de instruir y gobernar a los fieles, de tal modo que ellos mismos, también con la ayuda de estos medios, alcancen la salvación y la perfección propias y de todo el género humano (Inter Mirifica, 2000: 3).

Todavía no se hablaba de Internet, sin embargo, ya se están dando pasos importantes en relación con el "derecho originario que tiene la Iglesia de utilizar y poseer toda clase de medios [de comunicación] en cuanto que sean necesarios y útiles para la educación cristiana y para toda su labor de salvación" (Inter Mirifica, 2000: 3).

Pero ya en los últimos años del siglo XX, se va oficializando una enseñanza católica sobre el uso de Internet, enseñanza que ha ido en desarrollo continuo. Cinco años antes de que Internet llegara a ser una realidad para mucha gente, en 1990, el documento "El mensaje cristiano en la actual cultura informática", el papa Juan Pablo II pidió a la iglesia tener en cuenta los nuevos recursos logrados por la exploración humana en computarización y tecnología satelital para atender a la tarea de la evangelización (Juan Pablo II, 1990).

En 1995 comenzó el Website del Vaticano. En el 2001 el mismo Papa habló de las capacidades positivas de Internet de llevar información y enseñanzas religiosas más allá de todas las barreras y fronteras (Juan Pablo II, 2001). Al año siguiente en el mensaje "Internet, un nuevo foro para la proclamación del evangelio" afirmó que el nuevo mundo del ciberespacio es una gran posibilidad para la gran aventura de proclamar el mensaje evangélico, pero previno de las relaciones mediadas electrónicamente 
nunca pueden tomar el lugar del contacto humano directo que requiere la evangelización (Juan Pablo II, 2002).

Bajo el pontificado de Benedicto XVI se fue extendiendo la presencia del Vaticano en Internet a través del canal de televisión en Youtube en 2008 y del sitio Pope2You en Facebook en 2009. En este mismo año el Papa llamó a evangelizar el continente digital y llegar a ser heraldos del evangelio en la nueva ágora que se está abriendo (Benedicto XVI, 2009). Al respecto, los sacerdotes deben ser líderes que se expresen con las diferentes voces que provee el mercado digital utilizando los recursos audiovisuales como imágenes, videos, filmes animados, blogs, websites, entre otros, de manera que pueden abrir nuevos caminos para el diálogo, la evangelización y la catequesis (Benedicto XVI, 2010). En mensajes posteriores el Papa llama la atención sobre los perfiles digitales de la gente en las redes sociales como una forma de testimonio puesto que no basta insertar contenido religioso expreso en las nueva media, sino también testimoniar consistentemente en el propio perfil digital de cada uno en cuanto opciones, preferencias y juicios que sean consistentes con el evangelio aunque no se hable de ello específicamente (Benedicto XVI, 2011).

El Papa Francisco (2014) en su mensaje "Comunicación al Servicio de una auténtica cultura del encuentro" invita a no tener miedo de convertirse en ciudadanos del mundo digital pues el uso de las nuevas tecnologías de la comunicación como las redes sociales o Internet es legítimo, pero advierte que hay algunos peligros como la exclusión y la manipulación. Los medios pueden ayudar a entender un renovado sentido de unidad de la familia humana que nos impulsa a la solidaridad y el compromiso serio de una vida mejor para todos. Los medios de comunicación pueden ayudar en esta tarea, sobre todo hoy, cuando las redes de comunicación humana han alcanzado niveles sin precedentes de desarrollo. Abrir las puertas de las iglesias significa abrirlas asimismo en el mundo digital, tanto para que la gente entre, en cualquier condición de vida en la que se encuentre, como para que el Evangelio pueda cruzar el umbral del templo y salir al encuentro de todos.

De acuerdo con lo anterior, la Teología como disciplina se ve impulsada a una permanente renovación especialmente en sus métodos; con todo, no es el método lo único que en el campo teológico debe abrirse a los nuevos desafíos y horizontes que se abren hoy al espíritu humano, también requiere de la suficiente apertura para encontrar la manera de incorporar a su discurso el sentido profundo de los nuevos escenarios y ambientes donde se dan cita millones de creyentes para realizar múltiples actividades: el ciberespacio, entendido como el lugar virtual de encuentro hoy; encuentro de personas, de grupos de toda índole, pero también el 
espacio donde convergen finalmente la mayoría de los medios de comunicación.

Conscientes de este fenómeno, los Obispos latinoamericanos reunidos en Aparecida (Brasil) en la V Conferencia General del Episcopado latinoamericano, declaran:

La revolución tecnológica y los procesos de globalización conforman el mundo actual como una gran cultura mediática. Esto implica una capacidad para reconocer los nuevos lenguajes, que pueden ayudar a una mayor humanización global. Estos nuevos lenguajes configuran un elemento articulador de los cambios en la sociedad (CELAM, 2007: 484).

En razón de los nuevos lenguajes, los obispos latinoamericanos se comprometieron, entre otras cosas, a formar discípulos y misioneros que sean verdaderos "comunicadores profesionales competentes y comprometidos con los valores humanos y cristianos en la transformación evangélica de la sociedad" y a "estar presente en los medios de comunicación social: prensa, radio y TV, cine digital, sitios de Internet, foros y tantos otros sistemas para introducir en ellos el misterio de Cristo" (CELAM, 2007: 486).

Específicamente sobre Internet, la V Conferencia declara que este medio, dentro del panorama de la comunicación social, debe ser entendida en la línea ya proclamada en el Concilio Vaticano II como una de las "maravillosas invenciones de la técnica" (CELAM, 2007: 497). Acogiendo las palabras de Juan Pablo II, los obispos latinoamericanos subrayan: "La Iglesia se acerca a este nuevo medio con realismo y confianza. Como los otros instrumentos de comunicación, él es un medio y no un fin en sí mismo. La Internet puede ofrecer magníficas oportunidades de evangelización, si es usada con competencia y una clara conciencia de sus fortalezas y debilidades" (CELAM, 2007: 487).

En consonancia con esta visión general sobre Internet, hay que tener en cuenta dos aspectos importantes: la dimensión técnica de la virtualidad que requiere destrezas y competencias para el ambiente virtual y, la comprensión de la cultura y el espacio virtuales para llegar allí con la experiencia de la fe. Ciertamente, la pastoral va más allá de la simple aplicación de Internet como artefacto o como medio, es necesario que se asuma la virtualidad como lenguaje, ambiente, ágora, comunidad, conexión, donde se gesta una nueva cultura que exige de los creyentes una nueva manera de pensar y de comunicarse para poder transmitir su búsqueda espiritual y hacerla creíble y seria para otras personas y comunidades. 


\section{CiBERCULTURA}

Se puede definir la cibercultura como la cultura propia del mundo contemporáneo en el cual las tecnologías digitales presentan nuevas formas de información, comunicación, transmisión de experiencias, investigación, formación, manejo organizacional, seguridad, producción, etc. Esas nuevas formas van creando una cultura de la rapidez, la inmediatez, la telepresencia, que van conformando nuevos símbolos, valores, sentidos y lenguajes que conforman las nuevas subjetividades y las formas de encuentro y relación. Según Levy (2007), la cultura digital incluye lo simbólico, las técnicas, los entornos materiales, toda la información mediada por los dispositivos contemporáneos y los nuevos modos de pensamiento y de valores que surgen en el ciberespacio.

La cibercultura nos sitúa en un ambiente ecológico específico, donde se dan relaciones e interacciones distintas a las del pasado y con unos sentidos culturales nuevos en su formulación, pero humanos en su origen. Se hace un cambio respecto a la cultura agraria, industrial y letrada, para dar paso a una cultura nueva en sus formas de relación, de producción, de asociación y de intercambio de conocimientos. Se puede decir que cambian las formas de relación cara a cara, de la producción basada en trabajadores físicos presentes en las fábricas, y de la formación presencial en instituciones educativas, para dar paso a una relación virtual a través de las redes sociales, al teletrabajo, a la producción computarizada y a la formación virtual a distancia. Además, la cultura virtual lleva a la vivencia de la libertad casi total, lo que debilita el papel de control de las instituciones sociales.

Hablando críticamente, se podría decir que la relación y la presencia física, corporal, ceden el lugar a otro tipo de comunicación. Esto plantea problemas a la reflexión teológica y filosófica tales como la importancia de la presencia física para la interacción humana, el papel del cuerpo en la misma relación y el ejercicio de la autoridad en los grupos sociales.

El mundo de la virtualidad, como desarrollo y avance tecnológico y cultural es un medio que, aunque puede estar al servicio de la uniformización cultural, de la soledad y del consumismo, también tiene valores humanizadores. Se puede decir, que la red globaliza la conciencia crítica, social y ética, posibilitando diversas relaciones humanas. Como ya se va viendo, en la red no todo es uniformización, sino que se van conformando comunidades que son una especie de resistencia frente a lo homogéneo, transformándose en una expresión pública de proyectos de vida y acción. 


\section{CiBERTEOLOGÍA}

El desarrollo global de las TICs está generando esa nueva cultura (cibercultura) y provocando la apertura de la iglesia católica en particular y las diferentes iglesias en general al uso de esas tecnologías para realizar su labor.

En el caso de la iglesia católica se ha venido desarrollando una reflexión que incluye tres aspectos. En primer lugar, el uso de la red para colocar su mensaje como repositorios y bases de datos. Este uso simplemente aprovecha la capacidad de almacenamiento de la red para tener a la mano todos los documentos, mensajes y productos literarios de la iglesia. Es como un gigantesco repositorio de documentación, una biblioteca tradicional pero digitalizada. No se puede considerar directamente como teología de la red, sino como material para formación, investigación y memoria.

En segundo lugar, el uso de la red para evangelizar como metodología e instrumento. En este caso, los procesos que antes se hacían cara a cara, ahora se pueden hacer virtualmente. Se trata de transmitir mensajes, de hacer cursos de formación o catequesis, de comunicar noticias, eventos y actividades. Incluso para facilitar las labores burocráticas de las instituciones eclesiásticas tales como expedición de documentos, información de agendas y horarios, avisos de reuniones y retiros.

En tercer lugar, el uso de la red para construir una nueva reflexión teológica, como ciberteología para la cibercultura, está todavía en modo incipiente, pero sería la verdadera ciberteología o reflexión sobre la vivencia de la fe en y por la virtualidad.

Se trata de responder a la necesidad que tienen las diferentes confesiones religiosas de usar la virtualidad en sus procesos de publicidad, pastoreo, comunicación, pues las posibilidades y desarrollos de Internet son irreversibles y están conformando una nueva manera de vivir. Se hace imperiosa la necesidad de hacer la reflexión teológica sobre la cibercultura. Esa reflexión en campo católico, la hace notoria el sacerdote italiano Antonio Spadaro como una propuesta de (re)pensar la teología, entendida como inteligencia de la fe (intellectus fider), en el tiempo de la red y también de vivir la fe a partir de la lógica de la red digital. Spadaro (2014a) recoge las inquietudes ya existentes sobre el tema y las hace populares en su libro, Ciberteología: Pensar el cristianismo en tiempos de la red.

El objetivo de Spadaro es lanzar el desafío de hacer teología en la cibercultura comprendiendo sus potencialidades, lenguajes, medios, para poder vivir, expresar, comunicar y testimoniar la fe en el mundo digital. La cibercultura se convierte en el nuevo medio existencial para vivir la fe y el lugar para hacer la relación entre el Logos cristiano y la nueva cultura. 
Se trata de revelar al hombre el misterio del mismo hombre a partir de Jesucristo, pero en otras condiciones existenciales y técnicas.

La ciberteología es una reflexión apenas naciente. No hay claridad sobre su estatuto epistemológico, ni sobre su condición de teología regional, o simplemente si debe ser considerada como una metodología pastoral y no como teología. Pero, sin lugar a dudas, es un gran desafío si se tiene en cuenta que los jóvenes, los nativos digitales, ya van creciendo con otro lenguaje, otros conceptos existenciales, otras formas de comunicación y, la misma tecnología se va desarrollando frenéticamente hasta hablar de realidad virtual, comunicación por hologramas, presencia virtual, etc. Es necesario, por parte de la teología, hacer la interface entre fe y procesos comunicacionales. $\mathrm{Al}$ respecto, hay una abundante reflexión en las ciencias sociales tanto en América Latina, sobre todo en Brasil (Universidad Unisinos, por ejemplo) y en el ámbito anglosajón. Es un desafío difícil pues implica no sólo el conocimiento profundo de la cultura digital, sino también un cambio, una nueva manera de pensar, en la teología tal vez muy acostumbrada al libro, a la presencia física y al pastoreo territorial físico.

Las reflexiones sobre la cibercultura y su relación con la fe se han realizado principalmente en el campo antropológico cultural, etnográfico y religioso en general. En Europa se destaca Tim Hutchings (2012), sociólogo de la religión, los media y la cultura, que ha estudiado el impacto de los media sobre la religión. Su tesis titulada Creating Church Online: Ritual, Community, and New Media (Hutchings, 2010) presenta un trabajo centrado en el estudio sociológico de las Iglesias en línea, sobretodo en EE.UU, Australia e Inglaterra, mirando especialmente su forma de culto, el liderazgo y la relación comunitaria en ellas. Las Online churches (Iglesias en línea) son comunidades cristianas basadas en la red, que tienen su oración, su comunidad, y sus liturgias en Internet, y allí mismo buscan relaciones, amistades y dar a conocer su mensaje. Aunque comienzan a finales del siglo XX, hoy se han aumentado gracias a los avances en comunicaciones, dando origen a una nueva forma de vivir las creencias. En su investigación, analiza cinco diferentes iglesias online y las relaciones que establecen los creyentes a través de blogs, foros, chats, videos y mundos virtuales, mostrando cómo se dan allí las múltiples conexiones entre personas en un amplio espectro que va de la familiaridad a la diferencia y del aislamiento a la integración. Es un trabajo etnográfico o netnográfico, como lo proponen algunos, que aporta conocimientos al estudio de esta nueva forma religiosa.

En Brasil se destacan Pedro Gilberto Gomes (2010), Moisés Sbardelotto (2013) y Walter Avellar (2010) con trabajos más de tipo sociológico que ciberteológico. Sbardelotto (2016), en su tesis doctoral, hace un análisis de las nuevas formas de comunicación en Internet por parte de la 
iglesia católica. Estudia la forma de organizar los procesos de comunicación de la circulación católica en las redes de comunicación (Facebook y Twitter). El análisis se realiza a través de un estudio de casos múltiples desde un nivel suprainstitucional (la cuenta@Pontifex_pt, Twitter); a nivel institucional del Vaticano (Vaticano y Programa Brasileño en Facebook); a nivel socio-institucional de Brasil y a un nivel minoritario periférico de Brasil (la página Diversidad Católica en Facebook). En esta tesis, hay algunos elementos que indican características de ese nuevo mundo que son interesantes y que cuestionan al catolicismo: las interacciones producen nuevos discursos que van más allá de lo que ofrece la institución eclesial; esos discursos no son fijos sino que están sometidos a la interacción en la red; la interacción en la red es compleja e implica acciones sociales simbólicas; la circulación católica en la red conecta, une, forma comunidades e identidades, pero en forma plural se van formando nuevas conexiones; y así emerge una religión pública marcada por un poder simbólico compartido socialmente a través de la experimentación religiosa, cambiando el papel central de las instituciones de la iglesia en el establecimiento de las creencias y prácticas religiosas del entorno.

En Colombia se ha iniciado la reflexión con Fabián Salazar (2014) que, en su tesis de maestría, intenta mostrar las características generales que definen a la Internet y elabora una reflexión pastoral a partir de las categorías comunidad-comunicación-comunión y cómo debe acomodarse la iglesia para poder evangelizar en la red. En términos generales se encuentra mucha bibliografía sobre el uso eficaz y eficiente de los medios para la evangelización y para la pastoral virtual, pero todavía falta mucho en el tema de la reflexión propiamente ciberteológica.

En este sentido Susan George, se aproxima inicialmente a la ciberteología considerando cuatro posibles definiciones:

- Teología de los significados de la comunicación social en la era de Internet y de las tecnologías digitales, como una teología de la comunicación digital.

- Ponderación pastoral de la manera de anunciar el evangelio utilizando la red.

- Mapa fenomenológico de la presencia de lo religioso en Internet.

- Navegación en el ciberespacio en busca de las manifestaciones de lo espiritual (George, 2006: 182).

Como se puede apreciar, ninguna de estas definiciones llega propiamente a ser una teología del ciberespacio, sino una introducción al estudio de lo religioso virtual. Por su parte, otros autores, como Carlo Formenti 
(2000)definen ciberteología como una teología de la tecnología o de las connotaciones teológicas de la tecnociencias; por su parte, Debbie Herring, teóloga inglesa, aporta una clasificación muy útil al distinguir teología en el ciberespacio en cuanto colección de contenidos teológicos, teología del ciberespacio en el sentido de reflexiones teológicas para comprender la red y teología para el ciberespacio como compilación de sitios donde se pretende hacer teología en la red (Campbell \& Teusner, 2011).

En este recorrido de autores, se podría afirmar que Antonio Spadaro es quien se ha atrevido a formular un inicio de reflexión ciberteológica asumiendo el llamado de esa nueva cultura. La pregunta que se hace es "si las tecnologías digitales modifican el modo de comunicar y así mismo de pensar, ¿cuál es el impacto que tendrán en el modo de hacer teología?” (Spadaro, 2014a: 39). Así la ciberteología, según Spadaro (2011), se podría definir como una inteligencia de la fe en tiempos de la red o, pensar la fe a la luz de la lógica de la red, es decir, es la reflexión que surge de la pregunta sobre la forma en que la lógica de la Internet, con sus poderosas metáforas que trabajan en el imaginario, en la inteligencia, puede dar forma a la escucha y la lectura de la Biblia, la forma de entender la Iglesia y de la comunión eclesial, la Revelación, la liturgia, los sacramentos, es decir, los temas clásicos de la teología sistemática. La reflexión es importante, ya que resulta fácil ver cómo cada vez más el Internet contribuye a la construcción de la identidad religiosa de las personas, especialmente para la generación de los nativos digitales. La reflexión ciberteológica es siempre un conocimiento reflexivo desde la experiencia de la fe, siendo teología en el sentido de que responde a la fórmula Fides quaerens Intellectum, por lo tanto, no es Ciberteologia la reflexión sociológica sobre la religión en Internet, sino aquella que se refiere al fruto de la fe que se despliega cognitivamente en un momento en que la lógica de la red marca la manera de pensar, conocer, comunicar y vivir.

A su vez, la comunicación humana tiene un valor teológico pues ayuda a la Iglesia a entenderse a sí misma, por tanto, conocer su misterio. Además, la Iglesia puede ayudar a comprender la propia Internet ya que, al menos desde su propio punto de vista, la experiencia que vive el hombre en la red es la acción de Dios que mueve la humanidad hacia su plena realización. De este modo, Internet, con su capacidad de ser un espacio de comunión, es parte del camino del hombre hacia la plenitud de Cristo que llama a la humanidad a ser cada vez más unida y conectada. La ciberteología debe tener en cuenta que la cultura del ciberespacio coloca de manera objetiva, más allá de cualquier otra consideración, nuevos desafíos a nuestra capacidad para desarrollar y escuchar un lenguaje simbólico público que hable de la posibilidad y los signos de la trascendencia en nuestras vidas y de un mundo unificado y relacionado (Spadaro, 2011). 
Al respecto, Benedicto XVI (2011) afirmaba en su mensaje para la Jornada Mundial de las Comunicaciones que, cuando las personas intercambian información entre sí, ya están compartiéndose a sí mismos, su visión del mundo, sus esperanzas y sus ideales. La tecnología ayudando a crear una conexión de red, parece unir más estrechamente la amistad y el conocimiento, alentando a los hombres a ser testigos. De esta manera, el testimonio se está convirtiendo en la verdadera forma de comunicación en el entorno digital, por tanto, comunicarse significa ser testigo en el sentido de entender que la comunicación debe crear más humanidad.

La Iglesia está naturalmente presente allá donde el hombre desarrolla su capacidad de conocimiento y de relación. Sabemos, de hecho, como la Iglesia siempre ha tenido en el anuncio del mensaje y en las relaciones de comunión dos pilares fundadores de su ser. Así, el reto no debe ser la de cómo utilizar bien la red, como a menudo se cree, sino cómo vivir bien en el tiempo de la red. En este sentido, la red no es un nuevo medio de evangelización, sino de un contexto en el que la fe está llamada a ser expresada; esto no por un simple deseo de presencia, sino por una connaturalidad del cristianismo con las vidas de los hombres. Para Spadaro (2012), el único camino para hacer ciberteología es tener la experiencia de la fe y la experiencia de la red. Si falta alguna de las dos, no se puede hacer la reflexión. A su vez, el método ciberteológico posee cuatro pasos: experiencia personal de Dios y de la red, reflexión, acción y evaluación (Spadaro, 2014b).

Interesante en Spadaro (2014a) su consideración del concepto de Noosfera como una intuición genial de Pierre Teilhard de Chardin. Cristo aparece como el impulso inicial y la realización final de toda la humanidad. Esta progresivamente se va complejizando, integrando y unificándose. Se va pasando de la Biosfera o evolución biológica a la Noosfera o evolución intelectual y espiritual que llevará a la conciencia universal donde el punto Omega será su realización plena. La tecnosfera será un paso intermedio que permitirá la plena comunicación entre los humanos pues los conectará a todos, aunque ocasione un momento de crisis. Para Spadaro, la teología puede aportar mucho a la comprensión de esta evolución con sus categorías escatológicas y la consideración de Cristo como plenitud total de la humanidad. Todo será recapitulado en Cristo es la expresión de este final. La intuición de Teilhard radica en que la humanidad está en proceso de evolución hacia la plenitud en Cristo, y Spadaro propone sobre esa intuición una iglesia en red que permita construir relaciones significativas. Se va creando una conciencia humana más global, más espiritual, más incluyente, como signo de la progresiva realización del Reino de Dios. El aporte de los actuales desarrollos técnicos es ir creando la infraestructura compleja de una inteligencia colectiva o inteligencia conectiva que sirva de base a la circulación de ideas, datos, conocimientos, anhelos y esperanzas. 
Al respecto, Moisés Sbardelotto (2012), otro de los iniciadores en el Brasil de la ciberteología, enuncia algunos riesgos de esta reflexión. En primer lugar, presenta el peligro de la reducción de la ciberteología a lo técnico o maquinal, olvidando lo poético, lo artesanal, lo existencial y dejando de lado al maquinante (el que dirige la máquina). La ciberteología no puede ser una reducción de la teología al manejo técnico de los medios y programas. No es una dominación de lo técnico sobre lo religioso. Sería una ciberidolatría.

Otro riesgo es abstraer la vivencia religiosa de lo tecnológico de tal manera que se siga haciendo teología sin tener en cuenta la cultura digital. Es como un retiro del mundo para conservar lo religioso como no tocado o influenciado por lo tecnológico. El desafío de la teología es pensar a Dios en medio a las mediaciones humanas como la cultura digital, con sus luces y sombras. El peligro lo constituiría la desencarnación del mensaje cristiano, puesto que la cultura digital es un trabajo en red y complejo (network), constituido por relaciones vivas entre lo humano, la tecnología, el ambiente y también la teología.

Los riesgos enunciados por Sbardelotto (2012) se refieren a la actitud ante la tecnología, es decir, la aceptación acrítica o rechazo total, pero hay otros problemas más graves todavía con la incipiente ciberteología. Uno de ellos lo constituiría el problema de la sacramentalidad, pues no sólo coloca la cibercultura el problema de la presencia física en los rituales (eucaristía virtual, por ej.), sino la misma mediación de la iglesia como comunidad que se reúne, se encuentra y se comunica. ¿Será posible la ekkelesía virtual, la cyberchurch? ¿La comunidad virtual es legítima comunidad de seres humanos?

Otro, sería el problema de la autoridad. Siendo Internet un ámbito de libertad, ¿cómo podrán los pastores de las iglesias, guiar sus comunidades y mantener la ortodoxia y la ortopraxis?, ¿No dará ésto pie a la disgregación de las iglesias institucionales y a la multiplicación de grupos religiosos?

Finalmente, se podría vislumbrar el problema de la corporeidad. La red permite relaciones virtuales que indican un nuevo tipo de corporalidad no física. ¿Será posible y suficiente ese tipo de relación para la existencia humana y para la vivencia de la religiosidad?

4. LOS DESAFÍOS PARA LAS IGLESIAS EN EL CAMPO DE LO SOCIAL Y LO PÚBLICO

Las tendencias generales de la comunicación hacia el futuro pueden resumirse en el aumento de equipos portátiles interconectados con voz y audio inmediatos que permitan comunicación y respuesta instantánea y siempre más evolucionados; interconexión con personas, cosas (Internet 
de las cosas), instituciones, lo que favorecerá una mayor interacción y a mayor velocidad; la sociedad será más global y comunicada con oportunidades de interacción y de solidaridad universales.

Esto se dará en una sociedad culturalmente distinta, diversa, plural, secularizada, tecnológica, democrática, sensible a los derechos humanos, pragmática y experiencial. Esa sociedad planteará unos desafíos a la iglesia como son: globalización de la misma iglesia, relación con otras religiones, expansión del rol de los laicos, pérdida de poder de la jerarquía eclesiástica, pentecostalización de la experiencia religiosa, desafío de la revolución biotecnológica, multipolarismo político y religioso, mestizaje cultural y pérdida de monopolio religioso.

Concretamente, en el campo de la virtualidad, hay unos desafíos grandes para la iglesia en orden a responder a estas tendencias del futuro. Se precisarán algunos aspectos pastorales:

- De la pastoral de la respuesta a la pastoral de la pregunta. "Nunca hay que responder preguntas que nadie se hace" (Francisco, 2013: 55).

- De la pastoral centrada en los contenidos a la pastoral centrada en las personas: "la jerarquía eclesiástica, como también la política y social, debe encontrar nuevas formas para elaborar la propia comunicación, a fin de que su aporte a este fórum reciba una atención adecuada. Estamos aprendiendo a superar el modelo del púlpito y de la asamblea que escucha por el respeto de nuestra posición. Estamos obligados a expresarnos nosotros mismos de modo que involucremos y convenzamos a los otros que a su vez comparten nuestras ideas con sus amigos, followers o compañeros de diálogo" (Spadaro, 2014).

- De la pastoral de la transmisión a la pastoral del testimonio: "La misión no se limita a un programa o proyecto, sino que es compartir la experiencia del acontecimiento del encuentro con Cristo, testimoniarlo y anunciarlo de persona a persona, de comunidad a comunidad, y de la Iglesia a todos los confines del mundo" (CELAM, 2007:145), pero con otros lenguajes y conceptos.

- De la pastoral de la propaganda a la pastoral de la proximidad: "el desafío de descubrir y transmitir la mística de vivir juntos, de mezclarnos, de encontrarnos, de tomarnos de los brazos, de apoyarnos, de participar de esa marea algo caótica que puede convertirse en una verdadera experiencia de fraternidad, en una caravana solidaria, en una santa peregrinación” (Francisco, 2013: 87). 
- De la pastoral de las ideas a la pastoral de la narración: las redes sociales ofrecen la oportunidad de hacer más significativa la experiencia vivida subjetivamente gracias a las publicaciones, los videojuegos como medios narrativos, la transmisión de experiencias personales y locales y al compartir en una red global-local de relaciones.

- Una pastoral atenta a la interioridad y a la interactividad: ¿Es posible conjugar profundidad e interactividad? El desafío es muy grande. (Spadaro, 2014).

Todo lo anterior indica un proceso de cambio mental para las iglesias y la sociedad civil en cuanto exige:

- No considerar a Internet como un lugar de batalla para conseguir adeptos, sino el lugar del diálogo que armoniza las diferencias. El diálogo, que nace primero de la escucha, nos lleva a aprender a ver el mundo con ojos diferentes y a apreciar la experiencia humana tal como se manifiesta en las diversas culturas y tradiciones. Por tanto, dialogar significa estar convencido de que el otro tiene algo bueno para decir, dar espacio a su punto de vista y a sus propuestas. Dialogar no significa renunciar a las propias ideas y tradiciones, sino a la pretensión de que sean únicas y absolutas (Francisco, 2014).

- No confundir la acción evangelizadora con mercado de bienes religiosos, con la competencia religiosa, con el aumento de fieles, con búsqueda de rating, o con evangelizadores estrellas de la pantalla o de la red.

- La colaboración ecuménica, intercultural e interreligiosa puede fortalecer procesos de paz, de reconciliación, de reconstrucción del tejido social nacional, de solidaridad social. Incluso es el lugar para acciones conjuntas con gobiernos, sociedad civil y agencias de ayuda. Entender el ambiente de la red como lugar para construir la justicia y la paz en el mundo. "Internet puede ofrecer mayores posibilidades de encuentro y de solidaridad entre todos; y esto es algo bueno, es un don de Dios" (Francisco, 2014).

- Impulsar la investigación sobre las implicaciones sociales e institucionales de la práctica de la religión en línea y explorar qué impacto tendrá en la construcción de la identidad, de la comunidad, la autoridad y la autenticidad en la cultura más amplia. La cibercultura plantea importantes cuestiones éticas en relación con la autentici- 
dad, los límites de las responsabilidades privadas, públicas y políticas, y las de los individuos y las sociedades. Por lo tanto, los investigadores de la religión y los teólogos han de aumentar los estudios interdisciplinarios y fortalecer el diálogo académico. En este campo, el testimonio de la universidad católica en ese mundo, no es teórico, sino que debe reflejarse en una acción universitaria que se abra a todos: educación para todos, educación integral, respeto al pluralismo y a la libertad de conciencia, testimonio comunitario, libertad de investigación y diálogo fe-razón, cooperación y ayuda entre universidades católicas. Y aún más, repensar la forma de hacer teología en un gran esfuerzo de conjugar la tradición con la nueva cultura, y sirviéndose de una manera de pensar novedosa que privilegie la comunicación de la experiencia (Arboleda, 2014).

\section{CONCLUSIÓN}

La llamada ciberteología es un campo apenas en construcción, sin una epistemología ya fuerte y robusta. Es más, un desafío y un reto, que un producto logrado, ya que el mismo objeto de análisis, la cibercultura, es un proceso largo y complejo, del que no se puede decir la última palabra. La construcción de una reflexión seria sobre la nueva cultura será trabajo de largo aliento pues se han de sentar las bases: la primera de ellas es la discusión sobre temas claves como son la presencia corporal en el mundo digital, la comunidad en Internet como iglesia, el sentido y eficacia de la autoridad pastoral en la red, la sacramentalización y la reunión de manera virtual, el concepto de cuerpo y corporalidad, etc. Son todavía muchos los problemas y dificultades que se presentan en este campo. Los filósofos pueden ayudar a pensar si se puede comprender de otra manera la corporalidad, la presencia física, la comunidad, la comunicación interpersonal. Corrientes como el personalismo, la fenomenología, la ética humanista y la estética, pueden aportar mucho en este campo.

Un punto central y no obviable de una futura ciberteología es el estudio de la experiencia. No hay fe sin experiencia de Dios, pues toda religión parte de la experiencia de lo divino. Esta experiencia es múltiple: mística de la naturaleza, mística del encuentro, mística extática, mística de la belleza y el arte, mística social. ¿Puede el mundo digital ser lugar de la experiencia de lo divino? Es un problema a ser afrontado de manera teológica antes de internarse en los aspectos institucionales o sacramentales de la religión en Internet. Una nueva crítica de la razón mística para el mundo contemporáneo es esencial en este proceso, y el cristianismo tiene allí mucho que decir. 
La posibilidad de pensar teológicamente la intuición de Teilhard de Chardin de una evolución hacia la noosfera como meta en el punto omega cristológico, abre la reflexión sobre la construcción del Reino de Dios en medio de las dificultades producidas por las revoluciones de los últimos siglos, incluyendo allí la revolución de Internet.

En el campo de la ética, se puede notar que hay una mayor eficacia de la virtualidad pues las redes sociales permiten la solidaridad, la promoción de libertades y derechos humanos, la defensa de los oprimidos, la acción social global y las redes de resistencia contra las tiranías. Sin embargo, se presenta el aspecto oscuro de la red como explotación, delitos virtuales, promoción del terrorismo, falsas noticias. Urge allí la creación de una ética de Internet con medios adecuados y democráticos de control, formación y exigencia de responsabilidad.

Consecuencialmente, la pastoral ha de ir encaminándose a buscar los medios y el lenguaje más apropiados para el reto digital (Pontificio Consejo para las comunicaciones sociales, 2002). Muchos instrumentos que en el pasado fueron eficaces, hoy han de tomar nuevas formas para la comunicación abierta, dialogal, participativa y creativa. Hay un cierto miedo a lo digital ya que es un cambio cultural profundo. Pero hay que aprender esa nueva cultura, asimilarla críticamente y capacitar en lo digital. La red en sí no existe, existen personas que la pueden manejar y dirigir. El desarrollo de herramientas digitales y lenguaje apropiado con reflexión teológica adecuada, que permitan comunicar el mensaje de Jesucristo, es un imperativo pastoral de hoy.

\section{REFERENCIAS}

-Arboleda, C. (2014). Experiencia y testimonio. Medellín: UPB.

-Avellar, W. (2010). Internet e espiritualidade: o despertar através das mensagens de e-mail. Rio de Janeiro: Calibán.

-Bello, R. (2014). Las Tic al servicio de la evangelización. Recuperado de https://www.researchgate.net/publica-

tion/268504418_LAS_TIC_AL_SERVICIO_DE_LA_EVANGELIZACION_UNIVERSIDAD_CATOLICA_SANTO_DOMINGO

-Benedicto XVI (2009). Mensaje de su santidad Benedicto XVI, para la XLIII Jornada mundial de las comunicaciones sociales: Nuevas tecnologias, nuevas relaciones. Promover una cultura de respeto, de diálogo, de amistad. Recuperado de http://w2.vatican.va/content/benedict-xvi/es/messages/communications/documents/hf_ben-xvi_mes_20090124_43rd-world-communications-day.html

-Benedicto XVI (2010). Mensaje del S anto Padre Benedicto XVI, para la XLIV Jornada Mundial de las comunicaciones sociales: El sacerdote y la pastoral en el mundo digital: los 
nuevos medios al servicio de la Palabra. Recuperado de http:/ /w2.vatican.va/content/benedict-xvi/es/messages/communications/documents/hf_ben-

xvi_mes_20100124_44th-world-communications-day.html

-Benedicto XVI (2011). Mensaje del Santo Padre Benedicto XVI, para la XLV Jornada Mundial de las comunicaciones sociales: Verdad, anuncio y autenticidad de vida en la era digital. Recuperado de http://w2.vatican.va/content/benedict-xvi/es/messages/communications/documents/hf_ben-xvi_mes_20110124_45thworld-communications-day.html

-Campbell, H. \& Teusner, P. (2011). Religious Authority in the Age of the Internet. Recuperado de https://www.baylor.edu/content/services/document.php/130950.pdf

-CELAM (2007). Documento de Aparecida. São Paulo: Paulus.

-Formenti, C. (2000). Incantati dalla rete: Immaginari, utopie e conflitti nell'epoca di internet. Milano: Raffaello Cortina.

-Francisco (2013). Exhortación apostólica Evangelii Gaudium. Recuperado de http://www.vatican.va/evangelii-gaudium/po/index.html

-Francisco (2014). Mensaje del Santo Padre Francisco para la XLVIII Jornada mundial de las comunicaciones sociales: Comunicación al servicio de una auténtica cultura del encuentro. Recuperado de https://w2.vatican.va/content/francesco/es/messages/communications/documents/papa-francesco_20140124_messaggiocomunicazioni-sociali.html

-George, S. (2006). Religion and technology in the 21st Century: Faith in the e-World. Hershey: Information Science Publishing.

-Gomes, P. (2010). Da Igreja eletrônica à sociedade em midiatização. São Paulo: Paulinas.

-Hutchings, T. (2010). Creating Church Online: Ritual, Community, and New Media. Durham: Durham University.

-Hutchings, T. (2012). Network Theology: Christian Understandings of New Media. Journal of Religion, Media and Digital Culture 01(1), 1-14.

-Inter Mirifica (2000). Concilio Vaticano II. Madrid: BAC.

-Juan Pablo II (1997). Mensaje del Santo Padre para la XXXI jornada mundial para las comunicaciones sociales. Recuperado de http://w2.vatican.va/content/johnpaulii/es/messages/communications/documents/hf_jp-

ii_mes_24011997_world-communications-day.html.

-Juan Pablo II (2001). Discurso del santo padre Juan Pablo II a la academia pontificia de ciencias sociales. Recuperado de https://w2.vatican.va/content/john-paulii/es/speeches/2001/april/documents/hf_jp-ii_spe_20010427_pc-socialsciences.html

-Juan Pablo II (2002). Mensaje del Santo Padre para la XXXVI Jornada Mundial de las comunicaciones sociales: Internet, un nuevo foro para la proclamación del evangelio. Recuperado de http://w2.vatican.va/content/john-paul-ii/es/messages/communications/documents/hf_jp-ii_mes_20020122_world-communications-day.html 
-Juan Pablo II, (1990). XXIV Día Mundial de los Medios de Comunicación Social: El Mensaje cristiano en la actual cultura informática. Recuperado de http:/ /www.conferenciaepiscopal.nom.es/mcs/jornada/vaticano_90.htm

-Leone, M. (2014). Medium djihadiste et pensé fondamentaliste: un aperçu sémiotique. Recuperado de https://www.academia.edu/25837384/2016__Medium_djihadiste_et_pens $\% \mathrm{C} 3 \% \mathrm{~A} 9 \mathrm{e}$ _fondament-

liste_un_aper $\% \mathrm{C} 3 \% \mathrm{~A} 7 \mathrm{u} \_\mathrm{s} \% \mathrm{C} 3 \% \mathrm{~A} 9$ miotique

-Levy. P. (2007). Cibercultura. Informe al consejo de Europa. Barcelona: Anthropos

-Mason, P. (2016). Poscapitalismo: Hacia un nuevo futuro. Barcelona: Paidós Ibérica.

-Pontificio Consejo para las comunicaciones sociales (2002). Ética en Internet. Recuperado de http://www.vatican.va/roman_curia/pontifical_councils/pccs/documents/rc_pc_pccs_doc_20020228_ethics-internet_sp.html

-Salazar, F. (2014). Retos propuestos por Internet a la iglesia en el camino de la ciberevangelización. Medellín: UPB.

-Sbardelotto, M. (2012). Deus digital, religiosidade online, fiel conectado: estudos sobre religião e internet. Cadernos Teologia Pública (Ano IX, n. 70). São Leopoldo: IHU - Unisinos.

-Sbardelotto, M. (2013). "E o Verbo se fez bit": a comunicação e a experiência religiosas na internet. Aparecida: Santuário.

-Sbardelotto, M. (2016). "E o Verbo se fez rede": Uma análise da circulação e da reconstrução do "católico" na internet. Tesis de doctorado en ciencias de la comunicación. San Leopoldo: Universidade do Vale do Rio dos Sinos (Unisinos).

-Spadaro, A. (2011). Verso una "cyberteologia"? L'intelligenza della fede nel tempo della Rete. La Civiltà Cattolica, 1(3853), 15-27.

-Spadaro, A. (2012). A compatibilidade da fé à luz da lógica da rede. Recuperado de http://www.ihuonline.unisinos.br/index.php?option=com_content\&view $=$ article\&id $=4659 \&$ secao $=403$

-Spadaro, A. (2014a). Ciberteologia: Pensar el cristianismo en tiempos de la red. Barcelona: Herder.

-Spadaro, A. (2014b). Le 6 grandi sfide della comunicazione digitale alla pastorale. Recuperado de http://www.cyberteologia.it/2014/11/le-6-grandi-sfide-della-comunicazione-digitale-alla-pastorale/

Sumario: Introducción; 1. La orientación del Magisterio de la iglesia; 2. Cibercultura; 3. Ciberteología; 4. Los desafíos para las iglesias en el campo de lo social y lo público; Conclusión; Referencias. 